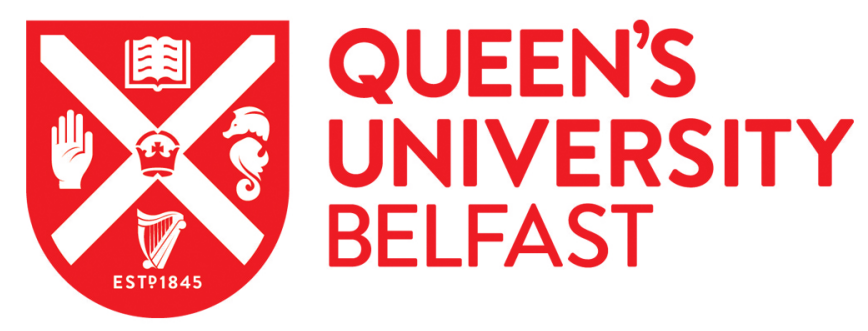

\title{
Ion-beam/plasma modes in ultradense relativistic quantum plasmas: Dispersion characteristics and beam-driven instability
}

Elkamash, I. S., Haas, F., \& Kourakis, I. (2017). lon-beam/plasma modes in ultradense relativistic quantum plasmas: Dispersion characteristics and beam-driven instability. Physics of Plasmas, 24. https://doi.org/10.1063/1.4989777

Published in:

Physics of Plasmas

Document Version:

Peer reviewed version

Queen's University Belfast - Research Portal:

Link to publication record in Queen's University Belfast Research Portal

Publisher rights

Copyright 2017 AIP Publishing. This work is made available online in accordance with the publisher's policies. Please refer to any applicable terms of use of the publisher.

\section{General rights}

Copyright for the publications made accessible via the Queen's University Belfast Research Portal is retained by the author(s) and / or other copyright owners and it is a condition of accessing these publications that users recognise and abide by the legal requirements associated with these rights.

Take down policy

The Research Portal is Queen's institutional repository that provides access to Queen's research output. Every effort has been made to ensure that content in the Research Portal does not infringe any person's rights, or applicable UK laws. If you discover content in the Research Portal that you believe breaches copyright or violates any law, please contact openaccess@qub.ac.uk. 


\title{
Ion-beam/plasma modes in ultradense relativistic quantum plasmas: dispersion characteristics and beam-driven instability
}

\author{
I. S. Elkamash ${ }^{1,2, *}$, F. $\operatorname{Haas}^{3 \dagger}$ and I. Kourakis ${ }^{1, \ddagger}$ \\ 1 Centre for Plasma Physics, Queen's University Belfast, \\ BT7 1NN Northern Ireland, UK \\ 2 Physics Department, Faculty of Science, \\ Mansoura University, 35516 Mansoura, Egypt \\ 3 Instituto de Física, \\ Universidade Federal do Rio Grande do Sul, \\ Av. Bento Gonçalves 9500, \\ CEP 91501-970, Porto Alegre, RS, Brazil
}

\begin{abstract}
A relativistic quantum-hydrodynamic plasma model is proposed, to model the propagation of electrostatic waves in an ultradense quantum electron-ion plasma in the presence of an ion beam. A dispersion relation is derived for harmonic waves and the stability of electrostatic wavepackets is investigated. Three types of waves are shown to exist, representing respectively a modified electron plasma (Langmuir-type) mode, a low-frequency ion acoustic mode, and an ion-beam driven mode. Stability analysis reveals the occurrence of an imaginary frequency part in three regions. The dependence of the instability growth rate on the ion beam parameters (concentration, speed) has been investigated.
\end{abstract}

\section{INTRODUCTION}

Beam-plasma interaction is an area of fundamental importance in the physics of charged matter [1-3], and also relevant in many real applications, such as heavy ion inertial fusion [4-6], intense laser-produced proton beams for laser based fast ignition (inertial confinement fusion) schemes [7-10], beam permeated semiconductor lasers [11-13] and electron cooling of ion beams [14, 15]. The excitation of electrostatic (ES) nonlinear localized waves $[16,17]$ via ion beam injection into plasma has been studied theoretically, via small-amplitude $[18,19]$ or largeamplitude nonlinear wave phenomenology [20] and also numerically, e.g. via particle-in-cell (PIC) simulations [21-25].

In ultra-high density and low temperature conditions, electrons obey a Fermi-Dirac statistics, hence the quantum pressure due to degeneracy effects largely dominates classical thermal pressure. Quantum degeneracy is relevant in a plasma when it is cooled to an extremely low temperature, so that the de Broglie wavelength associated with charge carriers becomes comparable in order of magnitude to intrinsic length scales, such as the mean interparticle distance and the Debye (screen) length. Quantum effects are arguably more relevant for electrons rather than ions, because of their lower mass. Degenerate plasmas are effectively studied via quantum-

\footnotetext{
*Email address: elkamashi@gmail.com

${ }^{\dagger}$ www.professor.ufrgs.br/fernando-haas; email address: fernando.haas@ufrgs.br

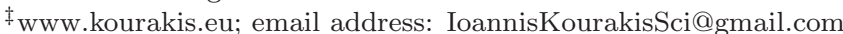

hydrodynamic models, incorporating an electron momentum equation which consistently takes into account the equation of state of the Fermi (degenerate) electron gas [26-28].

In this Letter, we investigate the existence of different propagating modes due to pumping positive ion beam into relativistic electron - ion plasma. The relativistic multifluid plasma model is introduced in the following section II. The dispersion characteristics of linear (harmonic) waves are described in Section III, and their dependence on the beam ion fluid features (number density and beam-fluid speed) are discussed. The stability analysis for different cases are investigated in section IV. Our findings are summarized in the concluding Section V.

\section{A RELATIVISTIC MULTIFLUID PLASMA MODEL}

We consider a three-component plasma consisting of a dominant ion population (mass $m_{i}$, positive charge $q_{i}=+Z_{i} e$ ), a secondary ion species, representing a tenuous beam (mass $m_{b}$, charge $q_{b}=+Z_{b} e$ ) and electrons (mass $m_{e}$, charge $-e$ ); $e$ denotes the elementary (absolute) charge, as usual. Spatial variation of the plasma plasma state variables is assumed to occur only in the longitudinal direction, hence the plasma dynamics can be described by a one-dimensional (1D) geometry for simplicity. Our study relies on a multifluid approach, to be introduced in the following paragraph. It is assumed from the outset that magnetic field generation may be neglected within the electrostatic approximation, implying that the total current is negligible (quiescent plasma). Our description follows closely the electrostatic relativis- 
tic model proposed in Refs. 29-30, thus extending the analytical framework proposed therein to take into account the ion beam.

The dominant (positive) ion population will be treated as a cold (classical) fluid, for simplicity; a plausible assumption, given their high mass (compared to the electrons). The continuity and momentum equations of motion for the ion fluid respectively read:

$$
\begin{aligned}
& \frac{\partial\left(\gamma_{i} n_{i}\right)}{\partial t}+\frac{\partial}{\partial x}\left(\gamma_{i} n_{i} u_{i}\right)=0, \\
& \frac{\partial\left(\gamma_{i} u_{i}\right)}{\partial t}+u_{i} \frac{\partial\left(\gamma_{i} u_{i}\right)}{\partial x}=-\frac{e Z_{i}}{m_{i}} \frac{\partial \phi}{\partial x},
\end{aligned}
$$

where $e$ is the electron charge, $Z_{i}$ is the ion charge state, $m_{i}$ is the ion mass, $n_{i}$ is the ion fluid density and $u_{i}$ is the ion fluid speed. One recognizes the electrostatic force $e Z_{i} E$ in the right-hand side (RHS) of the momentum equation, where $E=-\partial \phi / \partial x$ is the electric field deriving from an electrostatic potential function $\phi$.

The electron fluid equations read [29]:

$$
\begin{gathered}
\frac{\partial\left(\gamma_{e} n_{e}\right)}{\partial t}+\frac{\partial}{\partial x}\left(\gamma_{e} n_{e} u_{e}\right)=0 \\
\sqrt{1+\xi^{2}}\left[\frac{\partial\left(\gamma_{e} u_{e}\right)}{\partial t}+u_{e} \frac{\partial\left(\gamma_{e} n_{e}\right)}{\partial x}\right]= \\
\frac{e}{m_{e}} \frac{\partial \phi}{\partial x}-\frac{\gamma_{e}}{n_{e} m_{e}}\left(\frac{\partial P_{e}}{\partial x}+\frac{u_{e}}{c^{2}} \frac{\partial P_{e}}{\partial t}\right),
\end{gathered}
$$

where $m_{e}$ is the rest mass of the electron, $n_{e}$ is the electron fluid (number) density and $u_{e}$ is the electron fluid speed.

In ultrahigh density conditions, electron degeneracy effects become significant, and in fact far exceed the thermal pressure and, in very high densities, quantum pressure (expressed via a Bohm term [27]) too except for ultra-small wavelengths. The electrons then obey a Fermi-Dirac distribution, associated with an appropriate equation of state, which is incorporated in the model via the effective degeneracy pressure term in the highly relativistic limit, i.e. the last term in Eq. (4). Within our model, the quantum relativistic pressure term derives from the (1D) equation of state $[29,31]$ :

$$
P_{e}=\frac{2 m_{e}^{2} c^{3}}{h}\left[\xi\left(1+\xi^{2}\right)^{1 / 2}-\sinh ^{-1} \xi\right]
$$

where the parameter $\xi=p_{F e} / m_{e} c=h n_{e} /\left(4 m_{e} c\right)$ is related to the (high) electron density (note that the classical limit is recovered for $h \rightarrow 0$ ). One also distinguishes in the RHS of (4) the electrostatic force term, which relates the momentum equation to the electrostatic potential $\phi$.

The equations of motion for the ion beam read:

$$
\begin{aligned}
& \frac{\partial\left(\gamma_{b} n_{b}\right)}{\partial t}+\frac{\partial}{\partial x}\left(\gamma_{b} n_{b} u_{b}\right)=0, \\
& \frac{\partial\left(\gamma_{b} u_{b}\right)}{\partial t}+u_{b} \frac{\partial\left(\gamma_{b} u_{b}\right)}{\partial x}=-\frac{e Z_{b}}{m_{b}} \frac{\partial \phi}{\partial x},
\end{aligned}
$$

where $m_{b}$ is the beam ion mass, $n_{b}$ is the beam ion fluid density and $u_{b}$ is the beam ion fluid speed. The relativistic factor $\gamma_{j}=1 / \sqrt{1-u_{j}^{2} / c^{2}}$ (for $j=i, e, b$ ) appears in the fluid-dynamical equations, as a result of Lorentz transformations and resulting relations among different state variables between inertial frames. The equilibrium state (denoted by subscript ' 0 ') is defined as $\left.\left\{n_{i}, u_{i} ; n_{b}, u_{b} ; n_{e}, u_{e}\right\}\right|_{0}=\left\{n_{i 0}, 0 ; n_{b, 0}, u_{b 0} ; n_{e 0}, 0\right\}$, where $u_{b 0}$ is the unperturbed beam fluid speed.

The system is closed by Poisson's equation:

$$
\frac{\partial^{2} \phi}{\partial x^{2}}=\frac{e}{\epsilon_{0}}\left(\gamma_{e} n_{e}-\gamma_{i} Z_{i} n_{i}-\gamma_{b} Z_{b} n_{b}\right) .
$$

In the above relations, $c$ is the speed of light in vacuo, $h$ is Planck's constant, $\epsilon_{0}$ is the permittivity of free space and $e$ is the fundamental unit of electric charge. The quasineutrality condition in his system can be written as follows: $n_{e 0}-Z_{i} n_{i 0}-\gamma_{b 0} Z_{b} n_{b 0}=0$, where $\gamma_{b 0}=1 / \sqrt{1-u_{b 0}^{2} / c^{2}}, n_{e 0}, n_{i 0}$ and $n_{b 0}$ are the unperturbed densities of the electron, ion and beam ion population(s), respectively.

The fluid model can be cast in a dimensionless form, by adopting a set of characteristic scales: $t \rightarrow \omega_{p i} t, x \rightarrow$ $\omega_{p i} x / c_{s}, n_{j} \rightarrow n_{j} / n_{j 0}, u_{j} \rightarrow u_{j} / c_{s}$ and $\phi \rightarrow e \phi / 2 E_{F e}$, for $j=i, e, b$, where $\omega_{p i}=\sqrt{Z_{i} e^{2} n_{e 0} / \epsilon_{0} m_{i}}$ is the unperturbed ion plasma frequency (i.e., in a beam-free $e$ $i$ plasma). Note that the potential scale $\left(2 E_{F e} / e\right)$ and the characteristic speed scale $c_{s}=\sqrt{2 Z_{i} E_{F e} / m_{i}}$ are determined as functions of the non relativistic electron Fermi energy $E_{F e}=p_{F e}^{2} / 2 m_{e}$ and the Fermi momentum $p_{F e}=h n_{e 0} / 4$; this, in turn, prescribes the length scale as $c_{s} / \omega_{p i}$.

The fluid equations take the form:

$$
\begin{aligned}
& \frac{\partial\left(\gamma_{i} n_{i}\right)}{\partial t}+\frac{\partial}{\partial x}\left(\gamma_{i} n_{i} u_{i}\right)=0, \\
& \frac{\partial\left(\gamma_{i} u_{i}\right)}{\partial t}+u_{i} \frac{\partial\left(\gamma_{i} u_{i}\right)}{\partial x}=-\frac{\partial \phi}{\partial x}, \\
& \frac{\partial\left(\gamma_{e} n_{e}\right)}{\partial t}+\frac{\partial}{\partial x}\left(\gamma_{e} n_{e} u_{e}\right)=0, \\
& H\left[\frac{\partial\left(\gamma_{e} u_{e}\right)}{\partial t}+u_{e} \frac{\partial\left(\gamma_{e} n_{e}\right)}{\partial x}\right]= \\
& \frac{1}{\mu_{e}} \frac{\partial \phi}{\partial x}-\frac{n_{e} \gamma_{e}}{H \mu_{e}}\left(\frac{\partial n_{e}}{\partial x}+\alpha u_{e} \frac{\partial n_{e}}{\partial t}\right), \\
& \frac{\partial\left(\gamma_{b} n_{b}\right)}{\partial t}+\frac{\partial}{\partial x}\left(\gamma_{b} n_{b} u_{b}\right)=0, \\
& \frac{\partial\left(\gamma_{b} u_{b}\right)}{\partial t}+u_{b} \frac{\partial\left(\gamma_{b} u_{b}\right)}{\partial x}=-\frac{1}{\mu_{b}} \frac{\partial \phi}{\partial x}, \\
& \frac{\partial^{2} \phi}{\partial x^{2}}=\gamma_{e} n_{e}-\beta \gamma_{i} n_{i}-\delta \gamma_{b} n_{b}
\end{aligned}
$$

where $H=\sqrt{1+\xi^{2}}$ represents the dimensionless enthalpy of the system [29], where $\xi=\frac{h n_{e}}{4 m_{e} c}$; the relativistic factor is redefined as $\gamma_{j}=1 / \sqrt{1-\alpha u_{j}^{2}}$, where $\alpha=c_{s}^{2} / c^{2}=\mu_{e} \xi_{0}^{2}$ and $\xi_{0}=p_{F e} /\left(m_{e} c\right)=h n_{e 0} /\left(4 m_{e} c\right)$. 
We have also introduced the ion-to-electron charge ratio $\beta=\frac{Z_{i} n_{i 0}}{n_{e 0}}=1-\gamma_{b 0} \delta$, where $\gamma_{b 0}$ is redefined as $\gamma_{b 0}=1 / \sqrt{1-\alpha U_{b 0}^{2}}, U_{b 0}=\frac{u_{b 0}}{c_{s}}$ is the normalized equilibrium velocity of the beam, the beam-to-electron charge density ratio $\delta=\frac{Z_{b} n_{b 0}}{n_{e 0}}$, the electron-to-ion mass ratio $\mu_{e}=\frac{m_{e}}{m_{i}}(\simeq 1 / 1836 \approx 0.0005 \ll 1)$ and the mass ratio $\mu_{b}=\frac{m_{b}}{m_{i}}$. Note that overall charge neutrality is assumed at equilibrium, imposing $\beta=1-\gamma_{b 0} \delta$. As a representative "textbook" situation, we shall henceforth consider a hydrogen plasma $\left(Z_{i}=1\right)$ and a tenuous beam, i.e. $\gamma_{b 0} \delta \ll 1$, with $\mu_{b} \sim 1$ throughout.

\section{LINEAR DISPERSION RELATION}

Assuming small-amplitude harmonic variations around equilibrium, we shall set $n_{i, e, b}=1+\tilde{n}_{i, e, b} e^{i(k x-\omega t)}, u_{i, e}=$ $\tilde{u}_{i, e} e^{i(k x-\omega t)}, u_{b}=U_{b 0}+\tilde{u}_{b} e^{i(k x-\omega t)}$ and $\phi=\tilde{\phi} e^{i(k x-\omega t)}$ ( + complex conjugate, understood everywhere), with the understanding that the tilde'd quantities are very small (compared to the corresponding characteristic scales, e.g. $\tilde{n}_{e} \ll 1$ and so forth). One thus obtains a linear (Cramer) system for the amplitudes, in the form:

$$
\begin{aligned}
& -\omega \tilde{n}_{i}+k \tilde{u}_{i}=0, \\
& -\omega \tilde{u}_{i}+k \tilde{\phi}=0, \\
& -\omega \tilde{n}_{e}+k \tilde{u}_{e}=0, \\
& -H_{0} \omega \tilde{u}_{e}-\frac{k}{\mu_{e}} \tilde{\phi}+\frac{k}{H_{0} \mu_{e}} \tilde{n}_{e}=0, \\
& -\omega\left(\gamma_{b 0} \tilde{n}_{b}+\gamma_{b 1}\right)+k\left(\gamma_{b 0} \tilde{u}_{b}+\gamma_{b 0} U_{b 0} \tilde{n}_{b}+U_{b 0} \gamma_{b 1}\right)=0, \\
& -\omega\left(\gamma_{b 0} \tilde{u}_{b}+U_{b 0} \gamma_{b 1}\right)+k U_{b 0}\left(\gamma_{b 0} \tilde{u}_{b}+U_{b 0} \gamma_{b 1}\right)+\frac{k}{\mu_{b}} \tilde{\phi}=0, \\
& -k^{2} \tilde{\phi}+\beta \tilde{n}_{i}-\tilde{n}_{e}+\delta\left(\gamma_{b 0} \tilde{n}_{b}+\gamma_{b 1}\right)=0,
\end{aligned}
$$

where $\gamma_{b 1}=\alpha U_{b 0} \gamma_{b 0}^{3} \tilde{u}_{b}$. We have defined the equilibrium value of the enthalpy as $H_{0}=\sqrt{1+\xi_{0}^{2}}$.

From the above equations, we get the perturbed density of the plasma species:

$$
\begin{aligned}
\tilde{n}_{i} & =\frac{k^{2}}{\omega^{2}} \tilde{\phi}, \quad \tilde{n}_{e}=-\frac{1}{\mu_{e} H_{0}} \frac{k^{2}}{\omega^{2}-\left(\frac{1}{\mu_{e} H_{0}^{2}}\right) k^{2}} \tilde{\phi}, \\
\gamma_{b 0} \tilde{n}_{b} & =\left(\frac{k^{2}}{\gamma_{b 0}^{2} \mu_{b}\left(\omega-k U_{b 0}\right)^{2}}\right) \tilde{\phi}-\gamma_{b 1} .
\end{aligned}
$$

Thus, the compatibility (vanishing determinant) condition leads to the requirement $\epsilon(\omega, k)=0$, where the plasma dielectric function reads:

$$
\begin{aligned}
\epsilon(\omega, k)=1 & +\chi_{i}(\omega, k)+\chi_{e}(\omega, k)+\chi_{b}(\omega, k), \\
=1 & -\frac{1-\delta}{\omega^{2}}-\frac{1}{\mu_{e} H_{0}} \frac{1}{\omega^{2}-\left(\frac{1}{\mu_{e} H_{0}^{2}}\right) k^{2}} \\
& -\frac{\delta}{\gamma_{b 0}^{2} \mu_{b}\left(\omega-k U_{b 0}\right)^{2}} .
\end{aligned}
$$

The definition of the dielectric susceptibility $\chi_{j}(\omega, k)$ of the three plasma components $(j=i, e, b)$ is evident in the RHS.

For arbitrary values of $U_{b 0}$, the exact general expression (18) must be taken into account. However, it may be argued, physically, that for $u_{b 0} \ll c$ (viz. $U_{b 0} \ll 1 \ll$ $\left.c / c_{s}\right)$, the beam ions may be treated classically, hence (18) reduces to

$$
\begin{aligned}
\epsilon(\omega, k)=1 & -\frac{1-\delta}{\omega^{2}}-\frac{1}{\mu_{e} H_{0}} \frac{1}{\omega^{2}-\left(\frac{1}{\mu_{e} H_{0}^{2}}\right) k^{2}} \\
& -\frac{\delta}{\mu_{b}\left(\omega-k U_{b 0}\right)^{2}} .
\end{aligned}
$$

Recovering dimensions, the general dispersion relation (18) reads:

$$
\begin{array}{r}
\frac{\omega_{p i}^{2}}{\omega^{2}}+\frac{\omega_{p e, r e l}^{2}}{\omega^{2}-\left(\frac{c \xi_{o}}{H_{0}}\right)^{2} k^{2}}+\frac{\omega_{p b}^{2}}{\gamma_{b 0}^{2}\left(\omega-k u_{b 0}\right)^{2}} \\
\approx \\
\frac{\omega_{p i}^{2}}{\omega^{2}}+\frac{\omega_{p e, r e l}^{2}}{\omega^{2}-\left(\frac{c \xi_{o}}{H_{0}}\right)^{2} k^{2}}+\frac{\omega_{p b}^{2}}{\left(\omega-k u_{b 0}\right)^{2}}=1,
\end{array}
$$

where we have used the (classical) definition of the plasma frequency $\omega_{p j}=\left[e^{2} Z_{j} n_{0 j} /\left(\epsilon_{0} m_{j}\right)\right]^{1 / 2}$ (for ions, electrons or ions beam, respectively, for $j=i, e, b)$; we have also defined the relativistic electron plasma frequency as: $\omega_{p e, r e l}=\omega_{p e} / \sqrt{H_{0}}$. (The non-relativistic limit $\gamma_{b 0} \approx 1$ was evoked in the last step).

One sees in the left-hand side (LHS) of (20) that the first term represents the background cold-ion plasma contribution which, in the absence of electrons and beam, would represent simple, non-propagating ion plasma osillations, say, $\omega_{i}=\omega_{p i}$. The second term is the electron contribution, incorporating the relativistic electron plasma frequency $\omega_{p e, r e l}$ (representing quantumrelativistic Langmuir waves, viz. $\omega_{e}^{2}=\frac{\omega_{p e}^{2}}{H_{0}}+\left(\frac{c \xi_{o}}{H_{0}}\right)^{2} k^{2}$ [30], should the other two species be neglected). Finally, the third term represents the beam, involving the beam plasma frequency $\omega_{p b}$ and the beam velocity $U_{b 0}$ : neglecting the other two components, this term would lead to a beam-driven beam mode, $\omega_{b}=k U_{b 0} \pm \omega_{p b}$ $[21,22,32]$. Qualitatively speaking, the above dispersion relation therefore represents a mixing between the three latter frequencies $\omega_{e, i, b}$, which are respectively modified due to interactions among them. The expected (electronion plasma) dispersion relation [29] - cf. (8) in Ref. 30 is recovered exactly in the absence of the beam, i.e. upon setting $\delta=0$ in Eq. (20):

$$
\omega^{4}-\left(\frac{\omega_{p e}^{2}}{H_{0}}+\omega_{p i}^{2}+\frac{\xi_{0}^{2}}{H_{0}^{2}} c^{2} k^{2}\right) \omega^{2}+\omega_{p i}^{2} \frac{c^{2} k^{2} \xi_{0}^{2}}{H_{0}^{2}}=0 .
$$

We have presented the dimensional form of the dispersion relation, in the above, for the sake of physical clarity and for future reference. Let us now revert to the dimensionless form of the dispersion relation, for ease of 
algebraic manipulation. The full dispersion relation resulting from Eq. (20) is a sixth-order polynomial in $\omega$, and may thus not be solved analytically. In order to gain some insight, one notices that Eq. (20) has six poles (singularities) at $\omega=0$ (double root), $\omega= \pm\left(c \xi_{0} / H_{0}\right) k$ and $\omega=U_{b 0} k$ (double root). Expanding the dispersion relation for linear electrostatic waves, one finds the polynomial form:

$$
\omega^{6}+c_{5} \omega^{5}+c_{4} \omega^{4}+c_{3} \omega^{3}+c_{2} \omega^{2}+c_{1} \omega+c_{0}=0 .
$$

where

$$
\begin{aligned}
& c_{0}= \frac{k^{4} U_{b 0}^{2} \beta}{H_{0}^{2} \mu_{e}}, \quad c_{1}=-\frac{2 k^{3} U_{b 0} \beta}{H_{0}^{2} \mu_{e}}, \\
& c_{2}= \frac{k^{2}}{H_{0}^{2} \mu_{e} \mu_{b}}\left\{-H_{0}^{2} U_{b 0}^{2} \beta \mu_{b} \mu_{e}+\delta\right. \\
&\left.+\mu_{b}\left[\beta-U_{b 0}^{2}\left(H_{0}+k^{2}\right)\right]\right\} \\
& c_{3}=2 k U_{b 0}\left[\beta+\frac{1}{\mu_{e} H_{0}^{2}}\left(H_{0}+k^{2}\right)\right] \\
& c_{4}=-\left[\frac{\delta+\beta \mu_{b}}{\mu_{b}}+\frac{1}{\mu_{e} H_{0}^{2}}\left(H_{0}+k^{2}\right)-k^{2} U_{b 0}^{2}\right], \\
& c_{5}=-2 k U_{b 0} .
\end{aligned}
$$

(Recall that $\beta=1-\delta$.)

First of all, upon setting $k=0$, we find the relation:

$$
\omega^{4}\left(\omega^{2}-\omega_{0, \delta}^{2}\right)=0
$$

where

$$
\omega_{0, \delta}^{2}=\left(1-\delta+\frac{\delta}{\mu_{b}}\right)+\frac{1}{\mu_{e} \sqrt{1+\xi_{0}^{2}}}
$$

A modification of the relativistic cutoff frequency [30] is evident, due to the beam (via $\delta$ ), but is practically negligible. For an indicative value of, say, $n_{0}=10^{11} \mathrm{~m}^{-1}$, one finds $\xi_{0}^{2} \simeq 0.0036$, hence $\sqrt{1+\xi_{0}^{2}} \simeq 1.0018$. Therefore, assuming say $\delta=0.1, \mu_{b}=1$ and $U_{b 0}=0.2$, we find that $\omega_{0, \delta}^{2} \simeq 1831$ (in the presence of the beam), while $\omega_{0, \delta=0}^{2} \simeq 1832$ in the absence of the beam. According to the latter relation (valid at $k=0$ ), we anticipate six (6) modes (solutions of the dispersion relation), four of which will pass from the origin, while the remaining two will satisfy $\omega= \pm \omega_{0, \delta}$ (as above), representing (a) modified electron plasma (Langmuir) branch(es).

We proceed by introducing $\omega=\omega_{r}+i \omega_{i}$ into equation (22), separating real from imaginary parts and then solving the resulting equations numerically, where $\omega_{r}$ and $\omega_{i}$ respectively represent the real part and the imaginary part (growth rate) of the frequency $\omega$. The procedure reveals the existence of six dispersion curves, which are arranged in pairs. Starting with the real parts $\omega_{j, r}$, a numerical investigation shows that these six curves are approximately symmetric around the $k$ - axis. In particular, we obtain:
- an electron plasma (Langmuir) mode, $\omega_{1, r}(k)$, depicted in Fig.1(c, d), accompanied by a negative mirror mode, say $\omega_{6, r}(k) \approx-\omega_{1, r}(k)$ (actually, with a relative numerical difference of $\approx 10^{-6}$ ),

- an ion-acoustic mode, $\omega_{2, r}(k)$, depicted in Fig.1(a, b), along with a negative mirror mode, say $\omega_{5, r}(k) \approx-\omega_{2}(k)$ (actually, with a relative numerical difference of $\approx 10^{-6}$ ),

and

- a beam-driven acoustic mode, $\omega_{3, r}(k)$, depicted in Fig.1(e, f); this practically overlaps with a sister mode, say, $\omega_{4, r}(k) \approx \omega_{3, r}(k)$.

A set of typical values $\left(n_{e 0}=10^{11} \mathrm{~m}^{-1}\right.$, or $\xi_{0}=$ $0.0604)$, and $\mu_{b}=1$, in account of a hydrogen ion beam) have been adopted in all of the plots.

Now, considering the imaginary parts, one finds that only the latter (beam-driven) mode possesses a finite imaginary part, with $\omega_{4, i}=-\omega_{3, i}$. From Fig.1(g,h), it is obvious that the growth rate (imaginary part) occurs in a finite window (in the wavenumber $k$ ) which extends to larger values as the beam velocity $U_{b 0}$ increases; the associated growth rate reaches a maximum value which remains practically constant, although it shifts to larger $k$ values, for higher $U_{b 0}$ : see Fig. $1 \mathrm{~g}$. However, as seen in Fig.1h, the maximum growth rate increases with an increase in the beam density $\delta$. Considering, for rigor, the beam-free case $(\delta=0)$, one finds that the beam-driven modes $\omega_{3,4}$ disappear, as expected, while the Langmuir and acoustic modes survive.

\section{COMPARISON WITH KINETIC THEORY}

To validate our results presented above, based on the fluid model, it would be interesting to compare with the results from the $1 \mathrm{D}$ relativistic Vlasov-Poisson system, which reads

$$
\begin{gathered}
\frac{\partial f_{s}}{\partial t}+\frac{p}{\Gamma_{s} m_{s}} \frac{\partial f_{s}}{\partial x}+q_{s} E \frac{\partial f_{s}}{\partial p}=0 \\
\frac{\partial E}{\partial x}=\frac{1}{\varepsilon_{0}} \sum_{s} \int q_{s} f_{s} d p
\end{gathered}
$$

where $s=e, i, b$ and $f_{s}=f_{s}(x, p, t)$ denote the phase space electron, ion and beam probability distribution functions and $\Gamma_{e, i, b}=\sqrt{1+p^{2} /\left(m_{e, i, b}^{2} c^{2}\right)}$. Assuming that the plasma is in equilibrium state with no electric field $E=0$ and each species has a distribution function

$$
f_{s}=f_{s 0}+f_{s 1}
$$

where $f_{s 0}=f_{s 0}(p)$ is the equilibrium distribution function, and $f_{s 1}=f_{s 1}(x, p, t)$ is a small perturbation associated with the small amplitude wave. As above, we neglect ion temperature effects, so that the equilibrium ion distribution function will be $f_{i 0}=n_{i 0} \delta(p)$. Also, the 


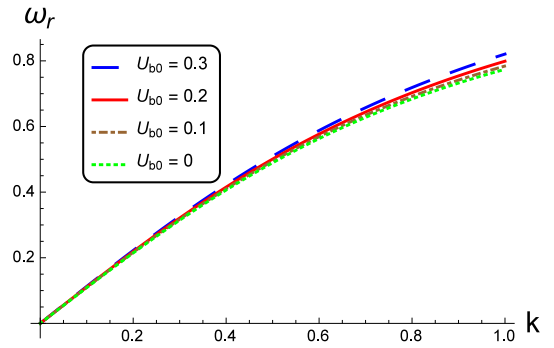

(a)

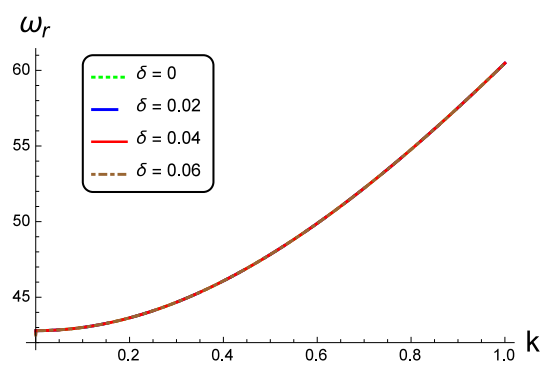

(d)

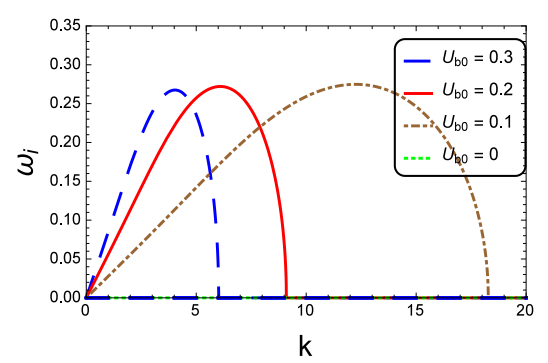

(g)

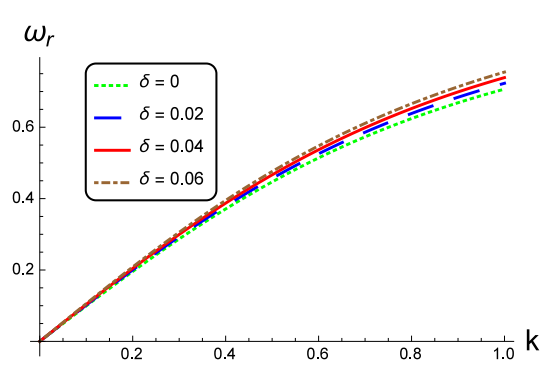

(b)

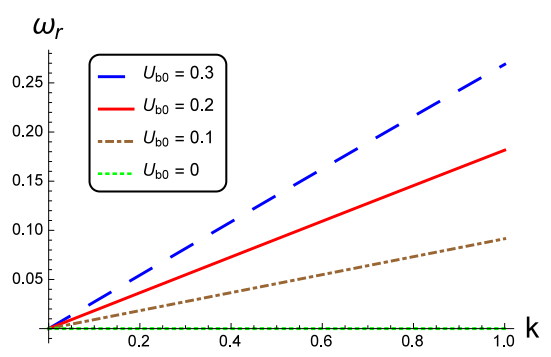

(e)

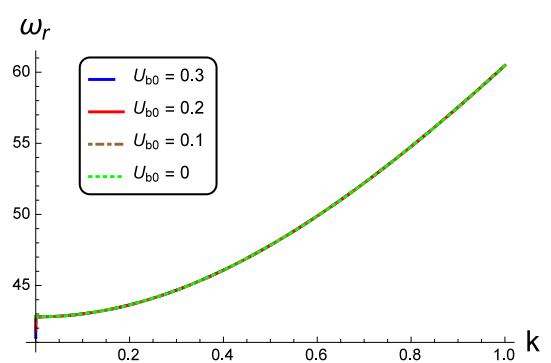

(c)

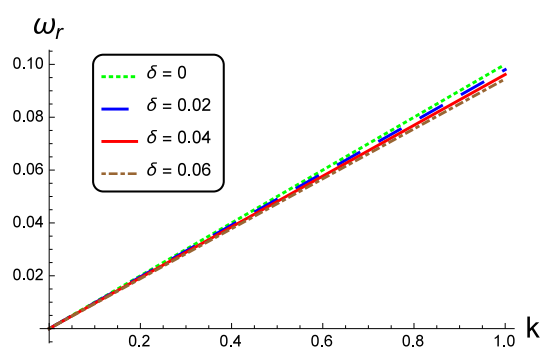

(f)

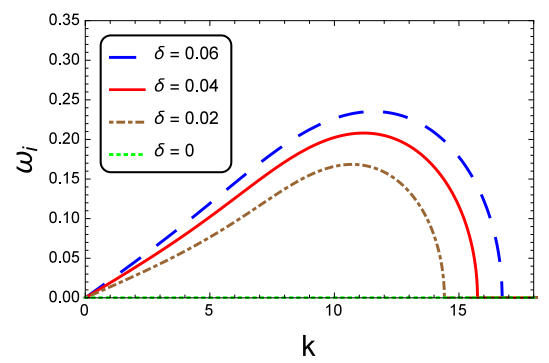

(h)

FIG. 1: (Color online) We have depicted the frequency $\omega$ versus the wave number $k$, for different values of the beam velocity $U_{b 0}$ (left column) and of the beam concentration $\delta$ (right column). The real part of the frequency $\omega_{r}$ is shown for: (panels a, b) the ion-acoustic branch; (panels c, d) the electron-plasma (Langmuir) mode; (panels e, f) the ion-beam driven branch. Panels $(\mathrm{g}, \mathrm{h})$ show the imaginary part (growth rate) $\omega_{i}$. We have considered $n_{e 0}=10^{11} \mathrm{~m}^{-1}$ (or $\xi_{0}=0.0604$ ) and $\mu_{b}=1$ (hydrogen ion beam) in all of the plots.

beam distribution will be $f_{b 0}=\Gamma_{b 0} n_{b 0} \delta\left(p-p_{0}\right)$, where $p_{0}=\Gamma_{b 0} m_{b} U_{b 0}$ and $\Gamma_{b 0}=\sqrt{1+p_{0}^{2} /\left(m_{b}^{2} c^{2}\right)}$. Denoting $f_{e 0}=n_{e 0} /\left(2 p_{F e}\right)$ for $|p|<p_{F e} ; f_{e 0}=0$ for $|p|>p_{F e}$ as the equilibrium electron distribution function, where $p_{F e}=\Gamma_{F} m_{e} V_{F e}$ and $V_{F e}$ is the electron Fermi speed, where $\Gamma_{F}=\sqrt{1+\xi_{0}^{2}}$. For each species $\int d p f_{s 0}(p)=n_{s 0}$, where $n_{s 0}$ is unperturbed number density of each species. By linearizing the above system and looking for plane wave solution $\exp (i(k x-\omega t))$, following the usual procedure [33], we get

$$
f_{s 1}(x, p, t)=\frac{-i q_{s} E}{\omega-\frac{k p}{\Gamma_{s} m_{s}}} \frac{\partial f_{s 0}(p)}{\partial p} .
$$

By inserting Eq. (29) into Eq. (27) and eliminate E from both side, we get

$$
1-\frac{e^{2}}{\varepsilon_{0} k} \int d p \sum_{s} \frac{\frac{\partial f_{s 0}(p)}{\partial p}}{\omega-\frac{k p}{\Gamma_{s} m_{s}}}=0,
$$

Upon integration by parts we get: 


$$
1-\int d p\left[\frac{\omega_{p i}^{2} f_{i 0}(p)}{n_{i 0} \Gamma_{i}^{3}\left(\omega-\frac{k p}{\Gamma_{i} m_{i}}\right)^{2}}+\frac{\omega_{p b}^{2} f_{b 0}(p)}{n_{b 0} \Gamma_{b}^{3}\left(\omega-\frac{k p}{\Gamma_{b} m_{b}}\right)^{2}}+\frac{\omega_{p e}^{2} f_{e 0}(p)}{n_{e 0} \Gamma_{e}^{3}\left(\omega-\frac{k p}{\Gamma_{e} m_{e}}\right)^{2}}\right]=0
$$

Using the equilibrium distribution function $f_{s 0}(p)$ and evaluate the real part of the integral, we get:

$$
1=\frac{\omega_{p i}^{2}}{\omega^{2}}+\frac{\omega_{p b}^{2}}{\Gamma_{b 0}^{2}\left(\omega-k U_{b 0}\right)^{2}}+\frac{\omega_{p e, r e l}^{2}}{\omega^{2}-\left(\frac{c \xi_{o}}{H_{0}}\right)^{2} k^{2}}
$$

which is exactly tantamount to (18) (upon restoring dimensions). It is understood that the imaginary part was ignored in evaluating the integrals. The contributions from the poles (known to be linked to collisionless "Landau-type" damping, as in the classical theory) may be evaluated for each of the modes, case by case. No general theory exists in this respect. We do not pursue this discussion further, as it goes beyond our scope here. An interested reader may refer to the discussion in Refs. $34-36$.

\section{STABILITY ANALYSIS}

To gain some insight into the stability profile of the dispersion relation, we may consider the behavior of the dispersion relation near the three poles of (19), thus defining respectively three frequency regions. The procedure we adopt here is similar to in Ref. 32 .

a. Low frequency instability. Near $\omega \approx 0$, the dispersion relation $\epsilon=0$ - recall Eq. (19) - becomes

$$
\frac{1-\delta}{\omega^{2}} \approx 1+\frac{H_{0}}{k^{2}}-\frac{\delta / \mu_{b}}{k^{2} U_{b 0}^{2}}
$$

It is straightforward to see that the RHS is positive, if (and only if) $U_{b 0}>\sqrt{\frac{\delta}{\mu_{b} H_{0}}}$, prescribing stability. However, if $U_{b 0}<\sqrt{\frac{\delta}{\mu_{b} H_{0}}}$, an instability develops at large wavelength: to see this, note that sufficiently small wavenumber values $k<\hat{k}_{10}=\left(\frac{\delta / \mu_{b}}{U_{b 0}^{2}}-H_{0}\right)^{1 / 2}$ will make the RHS negative. Typically, $\hat{k}_{10}=2.99$, for $\xi_{0}=0.06$, $\delta=0.1, \mu_{b}=1$ and $U_{b 0}=0.1$. The instability growth rate is then given by:

$$
\sigma_{1}=\operatorname{Im} \omega \approx\left(\frac{1-\delta}{\frac{\hat{k}_{10}^{2}}{k^{2}}-1}\right)^{1 / 2}
$$

We have depicted the growth rate $\sigma_{1}$ in Fig. 2, adopting the same values as in Fig.1.

It may be admitted, upon a critical comparison between the analytical approximation (34) and the numerical solution for $\omega_{i}$ in Fig. 3b, that there is a meagre

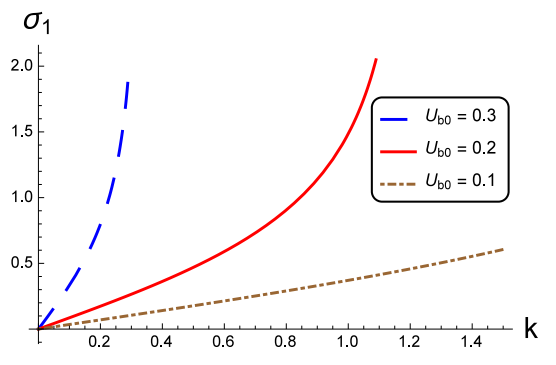

(a)

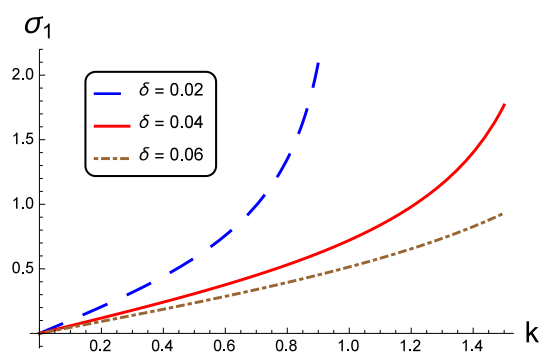

(b)

FIG. 2: (Color online) The growth rate $\sigma_{1}$, as given by Eq. (34), is depicted versus the wavenumber $k$ for (a) different beam velocity $\left(U_{b 0}\right)$ (taking $n_{b 0}=0.1 n_{e 0}$ i.e. $\delta=0.1$ ) (b) and different beam density $\left(n_{b 0}\right)$ (taking $U_{b 0}=0.1$ ) values. (Remaining parameter values as in Fig. 1.)

agreement in order of magnitude and, in fact, qualitative disagreement: the approximate expression for the growth rate (34) was derived above under the assumption that $\omega \approx 0$, and is thus only valid for very small values of $k$ (long wavelengths). In reality, the beam mode (see next paragraph) dominates in the small $k$ region, and indeed agrees with the numerical evaluation of the (imaginary part of) $\omega$, as discussed below. However, for small to moderate beam velocity, the ion-acoustic branch never satisfies the condition $\omega \approx 0$, under which the above expression (34) was obtained; therefore, Eq. (34), however important from a textbook analysis point of view (cf. Ref. 32), is of limited practical importance. Hence, the disagreement observed in Figs. 2 and 3 is not surprising.

b. Beam-wave resonance. Near $\omega \approx U_{b 0} k$, the wave's phase speed $\omega / k$ is near the beam velocity, $U_{b 0}$. The dispersion relation $\epsilon=0$ - recalling Eq. (19) - then becomes

$$
\left(\omega-k U_{b 0}\right)^{2} \approx \frac{\delta / \mu_{b}}{1-\frac{\hat{k}_{20}^{2}}{k^{2}}} .
$$




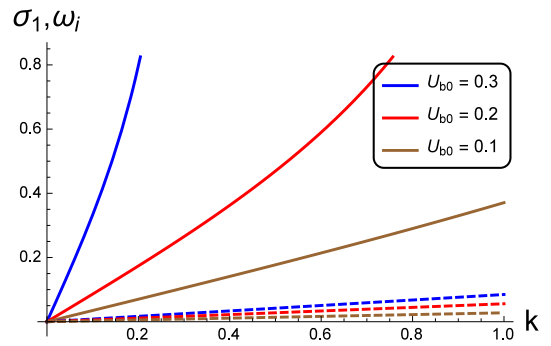

(a)

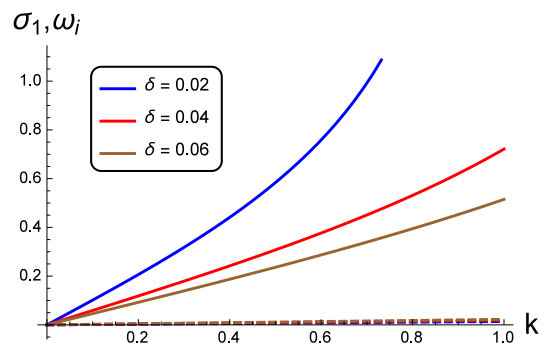

(b)

FIG. 3: (Color online) Comparison between the low-frequency instability growth rate $\sigma_{1}$ given by Eq. (34) (solid curve) and $\omega_{i}$ (dashed curve) versus the wavenumber $k$, for (a) different beam velocity $\left(U_{b 0}\right)$ (taking $n_{b 0}=0.1 n_{e 0}$ i.e. $\delta=0.1$ ) and (b) different beam density $\left(n_{b 0}\right)$ (taking $U_{b 0}=0.1$ ) values. (Remaining parameter values as in Fig. 1.)

where we have defined the quantity $\hat{k}_{20}=$ $\left[\frac{1}{\mu_{e} H_{0}} \frac{1}{U_{b 0}^{2}-1 /\left(\mu_{e} H_{0}^{2}\right)}+\frac{\beta}{U_{b 0}^{2}}\right]^{1 / 2} \cdot \quad$ As representative value, one finds $\hat{k}_{20}=9.43$, for $\xi_{0}=0.06, \delta=0.1, \mu_{b}=1$ and $U_{b 0}=0.1$ ). For large wavenumber values (short wavelength), viz. $\left(k^{2}>\hat{k}_{20}^{2}\right)$, one obtains a (stable) modified beam mode in the form:

$$
\omega \approx k U_{b 0} \pm \frac{\delta / \mu_{b}}{\left(1-\frac{\hat{k}_{20}^{2}}{k^{2}}\right)^{1 / 2}}
$$

However, if $k^{2}<\hat{k}_{20}^{2}$, then the RHS of Eq. (35) becomes complex, so that

$$
\omega \approx k U_{b 0} \pm i \sigma_{2},
$$

where $i=\sqrt{-1}$ is the fundamental imaginary number. An instability thus occurs, with growth rate

$$
\sigma_{2}=\left(\frac{\delta / \mu_{b}}{\frac{\hat{k}_{20}^{2}}{k^{2}}-1}\right)^{1 / 2}
$$

We have depicted the growth rate $\sigma_{2}$ in Fig. 4, for the same set of typical values as above. From Fig. 5, we can see that the numerical solution and the analytical approximation match well for small wavenumbers, in the region $k<\hat{k}_{20}$. Contrary to the previous case (for $\sigma_{1}$ ), a simple numerical analysis of the dispersion curves in Fig.

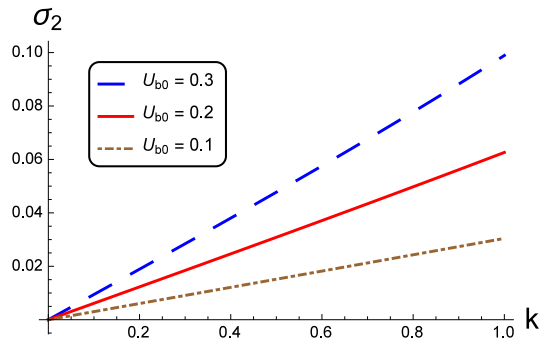

(a)

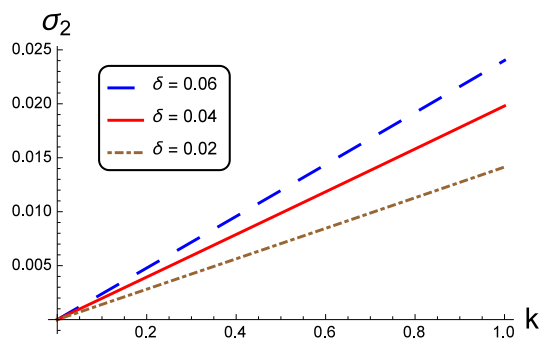

(b)

FIG. 4: (Color online) The growth rate $\sigma_{2}$ is depicted versus the wavenumber $k$ for (a) different beam velocity $\left(U_{b 0}\right)$ (taking $n_{b 0}=0.1 n_{e 0}$ i.e. $\left.\delta=0.1\right)$ and (b) different beam density $\left(n_{b 0}\right)$ (taking $U_{b 0}=0.1$ ) values. (Remaining parameter values as in Fig. 1.)

1 shows that the condition $\omega \approx U_{b 0} k$ under which Eq. (4) was obtained is indeed satisfied in some (small $k$ ) part of the beam-driven branch (only), hence the agreement observed in Figs 4 and 5 is expected.

c. High-frequency instability. In the vicinity of $\omega \approx$

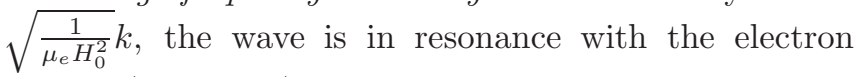
plasma (Langmuir) mode, since the phase speed is approximately equal to $\omega / k \approx \sqrt{\frac{1}{\mu_{e} H_{0}^{2}}}=\lim _{k \rightarrow \infty} \frac{\omega}{k}$; recall the dispersion relation for quantum - relativistic Langmuir waves. Equation (19) thus becomes

$$
\omega^{2} \approx \frac{1}{\mu_{e} H_{0}^{2}} k^{2}-\frac{\mu_{e} H_{0}}{\frac{\hat{k}_{30}^{2}}{k^{2}}-1}
$$

where $\hat{k}_{30}=\left((1-\delta) \mu_{e} H_{0}^{2}+\frac{\delta / \mu_{b}}{\frac{1}{\mu_{e} H_{0}^{2}}-U_{b 0}^{2}}\right)^{1 / 2} \cdot$ For $k>$ $\hat{k}_{30}$, the latter expression simply prescribes a modified Langmuir type dispersion relation of the form $\omega^{2}(k)=$ $\omega^{2}(0)+V^{2} k^{2}$, where $\omega^{2}(0)=\frac{\omega_{p e}^{2}}{H_{0}}$ and $V=\frac{c \xi_{0}}{H_{0}}$; for $\delta=0$, Eq. (25) in Ref. 30 is recovered.

For values of $k$ in the range $\hat{k}_{40}<k<\hat{k}_{30}$, where $\hat{k}_{40}^{2}=\left(\hat{k}_{30}^{2}-\mu_{e}^{2} H_{0}^{3}\right)^{1 / 2}$, the RHS of Eq.(39) becomes negative, so that

$$
\omega \approx \pm i \sigma_{3}
$$




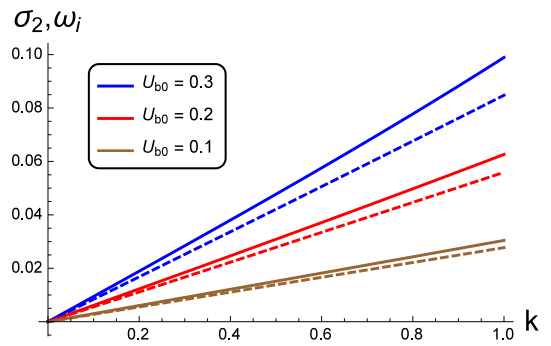

(a)

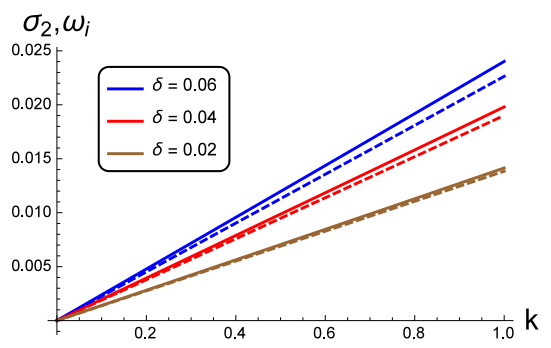

(b)

FIG. 5: (Color online) Comparison between $\sigma_{2}$ (solid curve) and $\omega_{i}$ (dashed curve) versus the wavenumber $k$, for (a) different beam velocity $U_{b 0}$ (taking $n_{b 0}=0.1 n_{e 0}$ i.e. $\delta=0.1$ ) and (b) different beam density $\left(n_{b 0}\right)$ (taking $U_{b 0}=0.1$ ) values. (Remaining parameter values as in Fig. 1.)

suggesting an instability growth rate in the form

$$
\sigma_{3}=\left(\frac{\mu_{e} H_{0}}{\frac{k_{30}^{2}}{k^{2}}-1}-\frac{1}{\mu_{e} H_{0}^{2}} k^{2}\right)^{1 / 2} .
$$

The instability window is actually very narrow; assuming $\xi_{0}=0.06, \delta=0.1, \mu_{b}=1$ and $U_{b 0}=0.1$, we find $\hat{k}_{30}=0.023397$ and $\hat{k}_{34}=0.023391$. However, the condition $\omega \approx \sqrt{\frac{1}{\mu_{e} H_{0}^{2}}} k(\approx 43 k$, say, for the numerical values considered in our plots) is only satisfied for the electron plasma branch, and exclusively for large $k \gg 1$; therefore, the latter result is not practically important, as it predicts an instability that is unlikely to develop for realistic beam values. We have therefore chosen not to depict the growth rate, for brevity.

d. Quantum (electron degeneracy) effect. The effect of electron degeneracy, intertwined with the relativistic nature of our model, is reflected in the parameter $\xi_{0}\left(n_{e 0}\right)$, that appears throughout the algebraic procedure presented above. In particular, the quantum character of our model is thus actually "hidden" in the dimensionless parameter $\xi=p_{F e} / m_{e} c=h n_{e} /\left(4 m_{e} c\right)$ appearing in Eq. (12) (via $H=\sqrt{1+\xi^{2}}$ therein) and, in fact, re-appearing as $\xi_{0}$ and $H_{0}$ (equilibrium values denoted by the index ' 0 ', as defined in the text) in Eq. (16), in the linear treatment. Therefore, the "quantum" effect is manifested, quantitatively speaking, in the value of $\xi_{0}=h n_{e, 0} /\left(4 m_{e} c\right)$, which is in fact proportional to the electron density $n_{e, 0}$, as intuitively expected: a higher density validates, and in fact imposes, the use of quantum
(Fermi-Dirac) statistics. (Naturally, the classical limit is recovered upon setting $\xi_{0}=0$, in account, say, of the limit $h \rightarrow 0$.)

In Fig. 6, we have depicted the dispersion relation derived above for different values of the electron density $n_{e 0}$. In Figs. 6(a, b), the frequency of both the ion acoustic branch and the Langmuir mode visibly decrease(s) with an increase in electron density $n_{e 0}$. The third branch (beam-driven mode) $\omega_{3}$ is shown in Figs. 6(c, e); as discussed above, it possesses an imaginary part, also shown in Figs. 6(d, f). Actually, the (real part of the) frequency $\omega_{3, r}$ is practically not sensitive to changes in the electron density: it does actually decrease, for higher values of $n_{e 0}$, but not dramatically: see Figs. 6(c, e). Actually, the growth rate $\omega_{3, i}$ slightly increases, for small $k$, with increasing $n_{e 0}$, while it then reaches a lower peak at higher $k$ : cf. $6(\mathrm{~d}, \mathrm{f})$.

\section{CONCLUSIONS}

We have presented a relativistic quantumhydrodynamic model for electrostatic waves propagating in an infinite homogenous quantum plasma permeated by a positive ion beam. From a physical point of view, relativistic effects arise from two sources: the extreme degeneracy of electrons producing a relativistic mass increase due to their incoherent motion, and relativistic beam speeds. Upon linearizing the models equations and subsequently Fourier analyzing, we have shown that three modes are excited, namely a modified Langmuir-type (electron plasma) mode, a low-frequency ion-acoustic mode and an ion-beam driven mode.

Numerical analysis of the dispersion relation reveals the occurrence of an imaginary part in the frequency $\omega$ that arises only in the beam-driven mode, and is entirely due to the beam itself (i.e. it disappears in the absence of the beam, viz. for $\delta \rightarrow 0)$. The standard textbook [32] stability analysis provides an approximate expression (see $\sigma_{2}$, above) for the imaginary part, which was shown to be a satisfactory approximation for small values of the wavenumber $k$ (very large wavelength). The dependence of the instability growth rate on the ion beam parameters has been investigated. Let us add, for completeness, that the same analysis allows for two more unstable regions; however, for small to moderate beam velocities, these were shown to be irrelevant practically for realistic applications.

The (quantum) effect of electron degeneracy on the electrostatic modes enters into play through the parameter $\xi_{0}\left(\sim h n_{e, 0}\right)$, as discussed in the previous section. Although electron degeneracy has a measurable effect on the ion-acoustic and Langmuir branches, with respect to the classical case (recovered for $h \rightarrow 0$ ), the beam-driven mode is marginally sensitive to changes in $\xi_{0}$ (modified by varying the electron density $\left.n_{e 0}\right)$. In any case, the frequency of all three modes in fact reduces for higher $\xi_{0}$, i.e. for higher density, as quantum effects become significant 


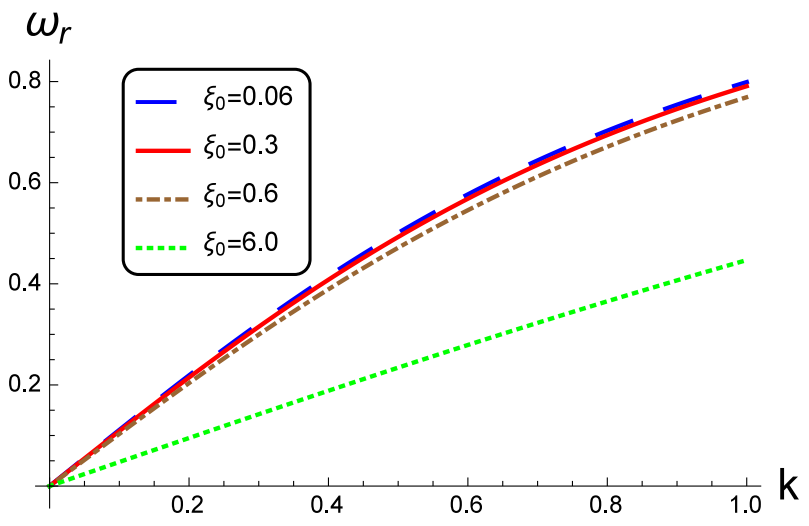

(a)

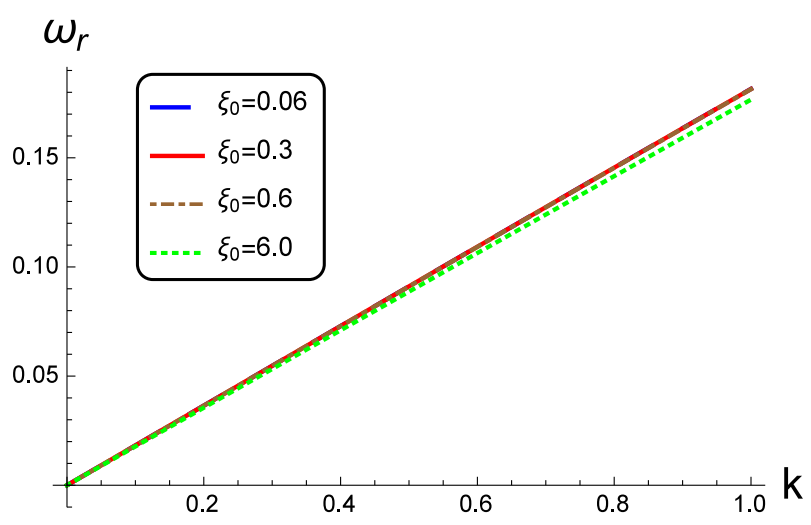

(c)

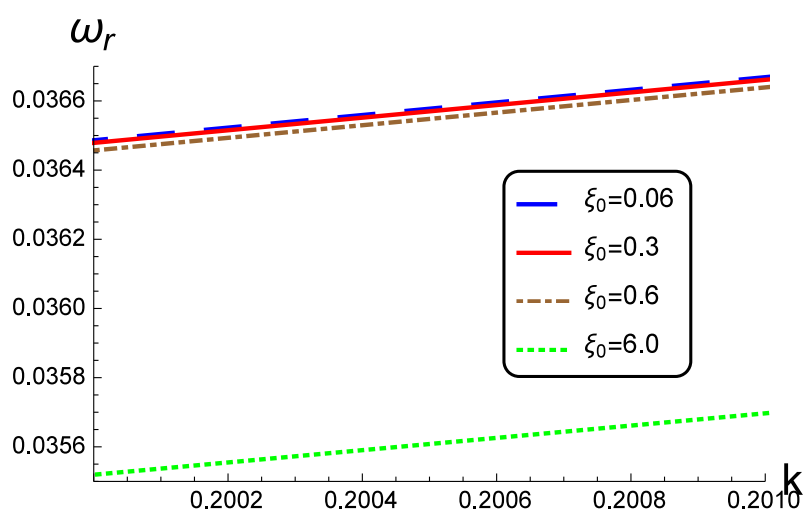

(e)

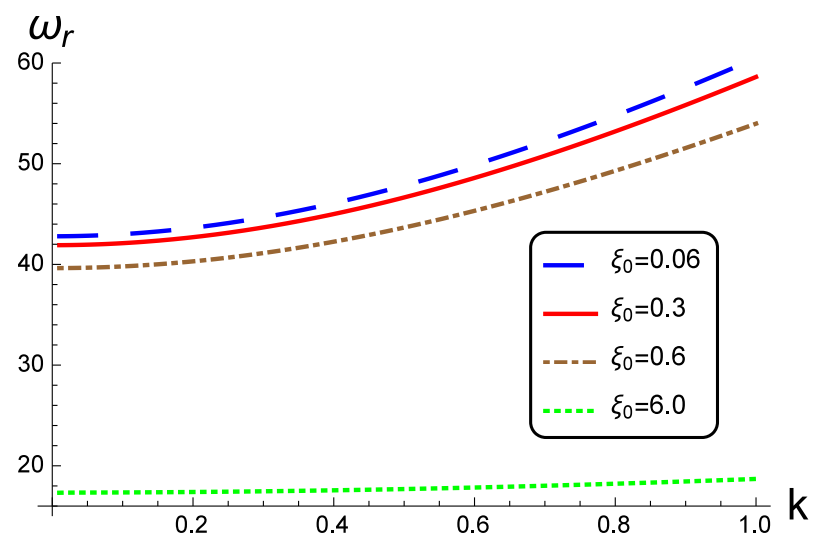

(b)

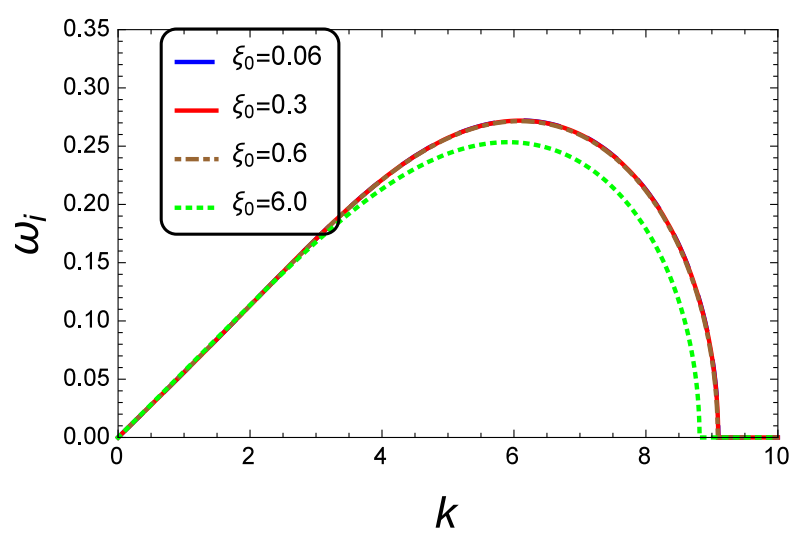

(d)

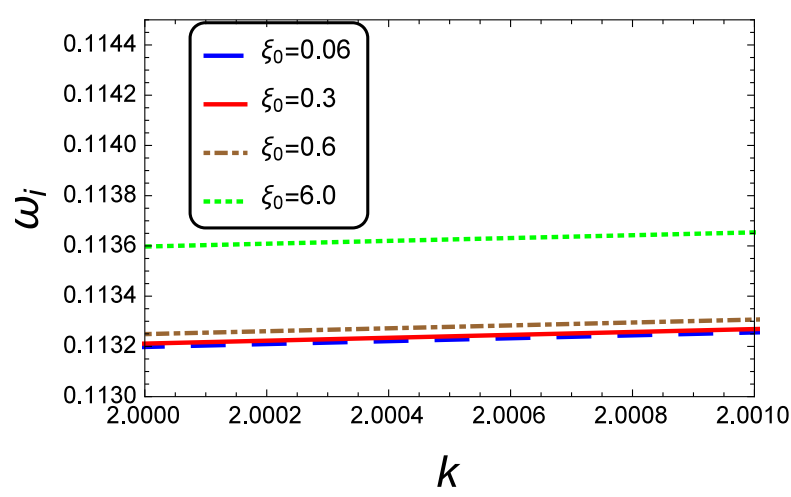

(f)

FIG. 6: (Color online) We have depicted the frequency $\omega$ versus the wave number $k$, for different values of relativistic degeneracy parameter $\xi_{0}$. The real part of the frequency $\omega_{r}$ is shown for: (panels a) the ion-acoustic branch; b) the electron-plasma (Langmuir) mode; c) the ion-beam driven branch; d) show the imaginary part (growth rate) $\omega_{i}$; e) Zoom - in for the ion-beam driven branch for the range $k=2: 2.001$ and f) Zoom - in for the imaginary part for the range $k=2: 2.001$. We have considered $U_{b 0}=0.2, \delta=0.1$ and $\mu_{b}=1$ (hydrogen ion beam) in all of the plots. The values of $\xi_{0}$ are: $\xi_{0} \approx 0.06,0.3,0.6,6.0$, respectively, for $n_{e 0}=10^{11}, 5 \times 10^{11}, 10^{12}, 10^{13} \mathrm{~m}^{-1}$, respectively. 
at larger density values. The imaginary part (beam instability growth rate) of the unstable beam-driven mode, however, increases for larger $\xi_{0}$.

Our results are important in quantum plasmas, dense plasmas arising from solid targets irradiated by high intensity laser and extreme astrophysical plasmas, that is, in areas where high-density plasma modelling is relevant.

\section{Acknowledgments}

The authors acknowledge support from the EU-FP7 IRSES Programme (grant 612506 QUANTUM PLAS-
MAS FP7-PEOPLE-2013-IRSES). FH and IK gratefully acknowledge support from the Brazilian research fund CNPq (Conselho Nacional de Desenvolvimento Científico e Tecnológico-Brasil). IK and I S Elkamash acknowledge the hospitality of Instituto de Física, Universidade Federal do Rio Grande do Sul (Porto Alegre, Brazil), where the largest part of this work was carried out. One of us (I S Elkamash) acknowledges financial support via an Egyptian government fellowship.
[1] R. B. Miller, An introduction to the physics of intense charged particle beams, Plenum Press (New York, 1982).

[2] Committee on High Energy Density Plasma Physics, Frontiers in High Energy Density Physics: The $X-$ Games of Contemporary Science, National Academic Press, 2003.

[3] R. P. Drake, High-Energy-Density Physics, SpringerVerlag (Berlin, 2006).

[4] C. M. Celata, F. M. Bieniosek, E. Henestroza, J.W. Kwan, E.P. Lee, G. Logan, L. Prost, P.A. Seidl, J.L. Vay, W.L. Waldron ,S.S. Yu, J.J. Barnard et al., Phys. Plasmas 10, 2064 (2003).

[5] W. M. Sharp, D. A. Callahan, M. Tabak, S. S. Yu, P. F. Peterson, D. R. Welch, D. V. Rose, and C. L. Olson, Fusion Science Technology 43, 393 (2003).

[6] R. C. Davidson, B. G. Logan, J. J. Barnard, F. M. Bieniosek, R. J. Briggs, D. A. Callahan, M. Kireeff Covo, C. M. Celata, R. H. Cohen, J. E. Coleman, C.S. Debonnel, D.P. Grote et al., Journal de Physique France 133, 731 (2006).

[7] K. Krushelnick, E. L. Clark, R. Allott, F. N. Beg, C. N. Danson, A. Machacek, V. Malka, Z. Najmudin, D. Neely, P. A. Norreys, M. R. Salvati, M. I. K. Santala, M. Tatarakis, I. Watts, M. Zepf, A. E. Dangor, IEEE Trans. Plasma Sci. 28, 1184 (2000).

[8] I. D. Kaganovich, E. A. Startsev, A. B. Sefkow and R. C. Davidson, Phys. Rev. Lett. 99, 235002 (2007).

[9] T. J. Renk, G. A. Mann, and G. A. Torres, Laser Particle Beams 26, 545 (2008).

[10] S. Ter-Avetisyan, M. Schnrer, R. Polster, P. V. Nickles, and W. Sandner, Laser Particle Beams, 26, 637 (2008).

[11] S. Colak, B. J. Fitzpatrick, and R. N. Bhargava, J. Cryst. Growth 72, 504 (1985).

[12] A. L. Gurskii, E. V. Lutsenko, A. I. Mitcovets, and G. P. Yablonskii, Physica B 185, 505 (1993).

[13] M. E. Yahia, I. M. Azzouz, and W. M. Moslem, Applied Physics Letters 103, 082105 (2013).

[14] A. H. Sorensen and E. Bonderup, Nucl. Instrum. Methods Phys. Res.215, 27 (1983).

[15] S. R. Goldman and I. Hofmann, IEEE Trans. Plasma Sci. 18, 789 (1990).
[16] E. Witt and W. Lotko, Phys. Fluids 26, 2176 (1983).

[17] Igor D. Kaganovich, Edward A. Startsev, and Ronald C. Davidson, Phys. Plasmas 11, 3546 (2004).

[18] W. M. Moslem, J. Plasma Phys. 61, 177 (1999).

[19] Esfandyari-Kalejahi A, I. Kourakis , B. Dasmalchi and M. Sayarizadeh, Phys. Plasmas 13, 042305 (2006).

[20] N. S. Saini and I. Kourakis, Plasma Phys. Control. Fusion 52, 075009 (2010).

[21] Q. Lu, S. Wang, and X. Dou, Phys. Plasmas 12, 072903 (2005).

[22] E.J. Koen, A. B. Collier, and S. K. Maharaj, Phys. Plasmas 19, 042101 (2012).

[23] M. E. Dieckmann, H. Ahmed, G. Sarri, D. Doria, I. Kourakis, L. Romagnani, M. Pohl and M. Borghesi, Phys. Plasmas 20, 042111 (2013).

[24] E.J. Koen, A. B. Collier, and S. K. Maharaj, Phys. Plasmas 21, 092105 (2014)

[25] K. Baumg'artel, Ann. Geophys. 32, 1025 (2014).

[26] D. Melrose, Quantum Plasmadynamics - Unmagnetized Plasmas, Springer-Verlag (New York, 2008).

[27] F. Haas, Quantum Plasmas: An Hydrodynamic Approach, Springer (New York, 2011).

[28] F. Haas, J. Plasma Physics, 82 (6), 705820602 (2016).

[29] M. McKerr, F. Haas, I. Kourakis, Physical Review E, 90, 033112 (2014).

[30] New insight in the dispersion characteristics of electrostatic waves in ultra-dense plasmas: electron degeneracy and relativistic effects, I. Kourakis, M. McKerr, I.S. Elkamash and F. Haas, submitted to Plasma Physics and Controlled Fusion (2017); accepted, in press.

[31] ] P. H. Chavanis, Phys. Rev. D 76, 023004 (2007).

[32] R. A. Treumann and W. Baumjohann, Advanced Space Plasma Physics, Imperial College Press (London, 1997).

[33] D Nicholson, Introduction to plasma theory, Cambridge Univ Press, 1983.

[34] A. E. Delsante and N. E. Frankel, Ann. Phys. 125, 137 (1980).

[35] J. Daligault, Phys. Plasmas 21, 040701 (2014).

[36] G. Brodin, J. Zamanian, and J. T. Mendonça, Phys. Scr. 90, 068020 (2015). 